\title{
Nonlinear Dynamic Phenomena in Macroscopic Tunneling
}

\author{
G. Dekel ${ }^{1}$, O.V. Farberovich ${ }^{2}$, A. Soffer ${ }^{3}$, V. Fleurov ${ }^{1}$ \\ ${ }^{1}$ Raymond and Beverly Sackler Faculty of Exact Sciences, School of Physics and Astronomy, \\ Tel-Aviv University, Tel-Aviv 69978 Israel. \\ 2 Department of Physics, Ben-Gurion University, Beer-Sheva 84105, Israel. \\ ${ }^{3}$ Department of Mathematics, Rutgers University, New Brunswick, NJ 08903, USA
}

\begin{abstract}
Numerical simulations of the NLSE (or GPE) are presented demonstrating emission of short pulses of the matter (light) density formed in the course of tunneling in wave-guided light and/or trapped BEC. The phenomenon is observed under various conditions, for nonlinearities of different signs, zero nonlinearity included. We study, both numerically and analytically, pulsations of matter (light) remaining within the trap and use the results in order to induce emission of sequential pulses by properly narrowing the trap. This allows us to propose a mechanism for a realization of Atom Pulse Laser.

Key words:

PACS: 82.20.Xr, 03.75.Kk, 05.90.+m
\end{abstract}

\section{Introduction}

The research on dynamics of systems described by Non Linear Schrödinger equation (NLSE), such as weakly interacting Bose - Einstein condensates (BEC) treated in the mean field approximation where it is known as Gross-Pitaevskii Equation (GPE), and light propagating in nonlinear refractive media, has generated many interesting results, most of which have to do with 'solitonic' behaviors of these systems. The realization of BEC in 1995 has made it possible to trace bright and dark matter wave solitons experimentally [12/344, and enhanced further vast theoretical work on the subject. The similarity between the two physical objects, optical systems and matter waves, both described by the similar equations, has made it possible to study both systems in parallel and shed light on one system by performing experiments on the other. Furthermore, the Atom Optics field has emerged, and very interesting applications of the optics principle implemented for matter waves were suggested and realized [5/6/7/89]. Interesting mechanisms for realization of pulsed Atom laser 
were also suggested. Refs. [10] and then [11] propose to use spatial variation of the scattering length so that the trapped condensate is accelerated towards a new minimum thus having a large enough energy to escape from the trap as a single soliton. Partial outcoupling is then achieved through an additional local maximum which causes splitting in the attractive interatomic interaction region, thus giving rise to a multiple emission. The authors also note that the velocities of the ejected solitons are constant and almost identical. Another mechanism was proposed in ref. [12] where an elongated, quasi 1d repulsive condensate is subject to simultaneous variations of the harmonic trap, from attractive to repulsive, and of the interatomic interaction, from repulsive to attractive, resulting in self coherent solitonic pulses. A special attention is given there to the stability criteria, mainly for avoidable collapse in the realistic multidimensional case. In refs. [1314] a periodic soliton array generator was introduced in the model that contained two tunnel-coupled parallel cigar-shaped traps, in which one trap is a BEC reservoir and the other trap is a lasing cavity.

Theoretical studies on dynamics of such systems have been carried out both numerically, perhaps the most common is the Split Step Fourier (SSF) method, and analytically, where some thorough and fruitful investigation were carried out [15|16[17]. Tunneling in one and two dimensional systems in the WKB approximation (with and without vortices) was addressed in the recent papers [1819. Behavior of BEC under the action of a time dependent field and tunneling through chaotic areas was analyzed in ref. [20. The hydrodynamic formulation of the Schrödinger equation was originally proposed in ref. 21] and has been in use since both in quantum mechanics and optics (see, e.g. 22 23|24|25|26]27]). An analytical study of macroscopic tunneling dynamics within the hydrodynamic representation of NLSE has been carried in Ref. 28129. This study employs the adiabatic approximation making use of the two characteristic time scales typical of the macroscopic tunneling processes 3013132 33, and results in an almost complete solution for the dynamics of tunneling of weakly interacting BEC. The most important feature of this solution is the observation of a tendency to formation of a dispersive shock wave in the outskirts of the potential trap, which propagates later on as a blip or pulse in the matter density or light intensity and which under certain conditions may form a bright soliton. It is important to emphasize that such pulses appear irrespective of the nonlinearity in the NLSE, and the show up, in particular, in the case of zero interaction (or zero Kerr nonlinearity in Optics).

Approaching BEC as a quantum fluid has recently given shock wave dynamic phenomena its rightfully deserved attention. Dispersive shock waves and related behaviors in BEC dynamics have been predicted theoretically and observed experimentally. In classical compressible gas and fluid dynamics, shock waves are known to be traveling fronts of steep gradients. However, since GPE admits no dissipation effects, the concept of dispersive shock waves has been introduced. $34|35| 36 \mid 37 / 38$ These are waves that are generated and maintained by dispersion instead of dissipation and are believed to consist of a number of spatial dark solitons and exhibit large amplitude oscillations.

In this work we further investigate the dynamics of 1 -d macroscopic tunneling by simulating temporary and spatial evolution of tunneling matter wave and light described within the frame work of NLSE/GPE. We carry out detailed simulations which demonstrate an agreement with formation and propagation of a blip [29] regardless of the type of nonlinearity. However, the latter causes differences in the further behavior of the outgoing pulse in the three cases, negative, zero and positive nonlinearity, which correspond to focusing, defocusing and 'nonfocusing' media in Optics, and respectively attractive, repulsive and zero interactions in BEC. 
We also demonstrate how different initial states rapidly transform into the state optimal for the blip formation used in Ref. 29. This indicates that the process of blip formation is not really sensitive to the initial conditions. We then concentrate on the matter (light) which remains trapped after the emergence of the blip, which is seen, both numerically and theoretically, to pulsate with the frequency mainly determined by the trap.

In the last part of this work we merge the two studied effects, i.e. single blip emission and periodic pulsations, to search for conditions under which blips may be repeatedly and controllably emitted. Such 'blip train' might pave a road for realization of Atom Pulsed Laser.(1011]12 13[14]) We show how each parameter of such train is controllable.

\section{Simulation}

Our simulation program is based on the Interaction Pictures Runge-Kutta-4 method 39] for solving the dimensionless GPE

$$
i \frac{\partial \Psi(x, t)}{\partial t}=\left[-\frac{1}{2} \nabla^{2}+U_{e x t}(x)+N U_{i n t}|\Psi(x, t)|^{2}\right] \Psi(x, t)
$$

where $\Psi(x, t)$ is the normalized to one wave function of the condensate with $N$ particles in it. The external potential

$$
U_{\text {ext }}=C \frac{1}{\cosh ^{2}\left(\frac{x}{a}\right)}\left(\frac{x}{b}\right)^{2} .
$$

is a symmetric tunnel trap (see Fig. 10) parameterized by the widths $a$ and $b$. $C$ is a constant which detemines the height $U_{0}=\max \left[U_{\text {ext }}(x)\right] \simeq 1$ fo the trap. $U_{\text {int }}$ is the effective interaction potential which may be positive (repulsive) or negative (attractive), or even zero. Our calculations use the values $\frac{N U_{i n t}}{U_{0}}= \pm 0.15,0$. Then the initial normalized Gaussian packet,

$$
\Psi_{0}(x)=\sqrt{\frac{1}{\sqrt{2 \pi} w}} \exp \left(-\frac{x^{2}}{4 w^{2}}\right),
$$

whose peak is situated at the center of the well at the time $t=0$, evolves dynamically according to the GPE equation (11). The parameters of the trap are $C=0.1$ (so that $U_{0}$ is close to one), $a=5, b=1$, and the width of the initial Gaussian input is $w=7$. Fig. 1 presents a sketch showing the trap and the initial packet.

\subsection{Application to Optics}

In parallel, another simulation program, the Free Beam Propagation Method (FBPM) [40], simulating optical pulses propagation in nonlinear media by solving the NLSE

$$
\frac{1}{2} \nabla^{2} A+\left(\frac{2 \pi}{\lambda}\right)^{2} \cdot n \cdot \Delta n(x) A+i\left(\frac{2 \pi n}{\lambda}\right) \frac{\partial A}{\partial z}+\left(\frac{2 \pi}{\lambda}\right)^{2} \cdot n \cdot n_{N L}|A|^{2} A=0
$$

was used. Here $A$ is the electric field amplitude in the paraxial approximation.

In order to get a full correspondence between Eq. (3) and the GPE (11), we considered the time independent process in the two dimensional $x, z$ space. The light beam propagates 


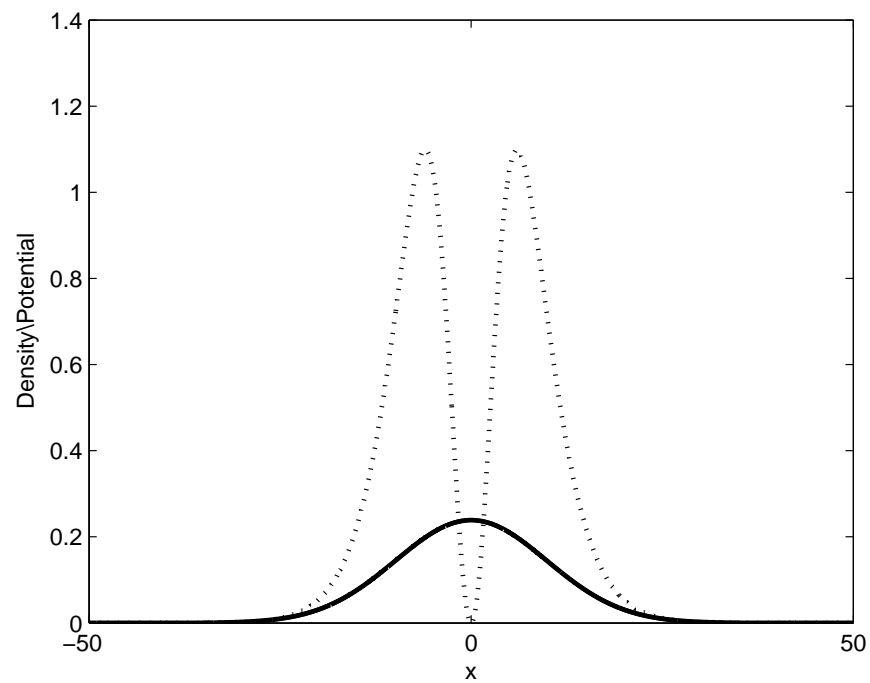

Fig. 1. The tunnel trap (dotted line) and initial packet (solid line) for the parameters $C=0.1, a=5$, and $b=1$, and $w=7$.

along the $z$ axis so that the $z$ coordinate plays the role of time in the GPE. $x$ is the 1-d traversal direction and corresponds to the space coordinate in the GPE. The 'potential trap' is actually a waveguide due to the variation of refractive index along the $x$ axis which can be fabricated by correspondingly varying the dopant concentration. The deviation $\Delta n$ of the refraction index from its global value has the form

$$
\Delta n(x)=\Delta n_{\max }\left(1-\frac{C}{\cosh ^{2}\left(\frac{x}{100}\right)} \cdot\left(\frac{x}{100}\right)^{2}\right)
$$

where we chose $C=2.28$ for the calculations. An initial Gaussian pulse centered at $x=0$, $z=0$ inside the trap is set to propagate in such a medium with the Kerr nonlinearity. The relevant medium (e.g., for AlGaAs) parameters are refractive index $n=3.33$ at $x \rightarrow \infty$, the Kerr nonlinearity $n_{2}=3 \cdot 10^{-16} \frac{\mathrm{m}}{\text { watt }}$, the maximal deviation of refractive index $\Delta n_{\max }=$ 0.001 , the waveguide width $W=2000 \mu \mathrm{m}$, and its length $L=35,000 \mu \mathrm{m}$. The initial input is a Gaussian shaped pulse of the width $w=300 \mu \mathrm{m}$ and intensities in the range 700 to 2000 watt. The wavelength is $\lambda=3 \mu m$.

\section{Results}

\subsection{Emergence of the Blip}

The simulation results for zero, negative and positive nonlinearities are presented in Fig. 2. We clearly see how a blip is created and propagates with a constant velocity independently of the nature of the nonlinearity, i.e. the blip shows up also in the case of zero or repulsive interaction in BEC, or zero or defocusing Kerr nonlinearity in optics. It surely exists in the 

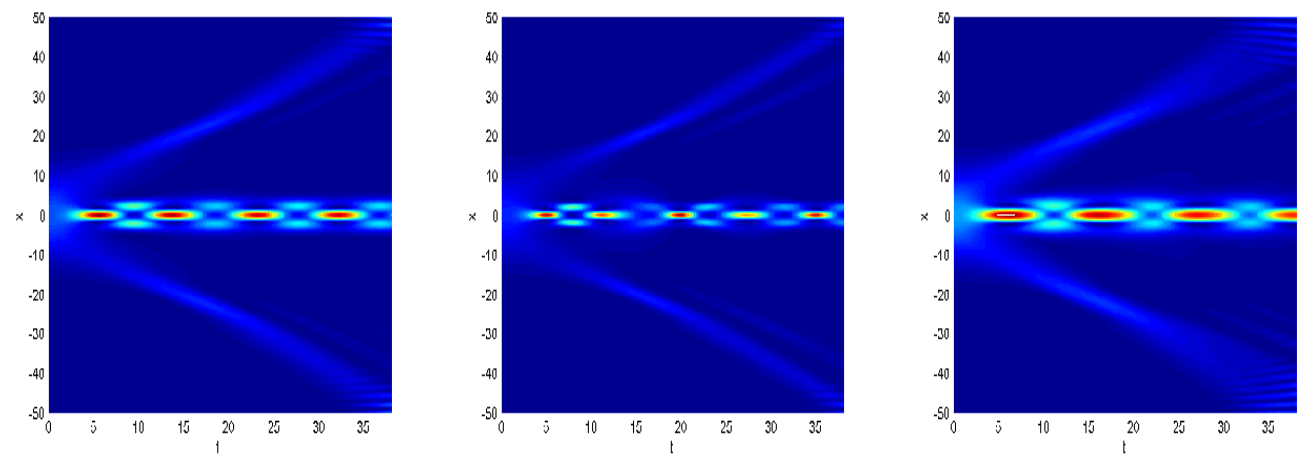

Fig. 2. Blip emergence and propagation. The figures show continuous top view onto the time evolution of BEC systems. From left to right: non focusing (zero interactions), focusing (attractive interactions) and de-focusing (repulsive interactions) cases . In the focusing case, the blip transforms into a bright soliton. It is also clear that the remaining trapped packet exhibit pulsations, a feature that will be discussed below.

negative nonlinearity case (see Fig. 2- focusing case), i.e. attractive interaction or focusing, in which case the blip under proper conditions may transform into a bright soliton. 29 .

Another universal feature of this phenomenon is the velocity of the blip propagation. As can be seen in Fig. 2 it moves with a constant velocity (along straight lines). Moreover, the velocity remains the same for different nonlinearities. Our analysis in Ref. [29] yields equation

$$
v_{b l i p}=v_{\text {shock }}=\sqrt{\frac{2 U_{0}}{m}}
$$

for the velocity of the dispersive shock wave propagation. Here $U_{0}$ is the height of the potential barrier in the BEC problem. In optics it is translated into the dimensionless quantity

$$
v_{b l i p}=\sqrt{\frac{2 \Delta n_{\max }}{n}} .
$$

We see an excellent agreement between the theory and simulations such as $v_{\text {theory }}=1.4$ and $v_{\text {simulation }}=1.3$ in the BEC case and $v_{\text {theory }}=2.45 e^{-2}=0.33$ and $v_{\text {simulation }}=2.57 e^{-2}=$ 0.35 in optics regardless of the type of nonlinearity.

As was outlined in our previous paper 29] the blip results from the tendency to a shock formation on the outskirts of the trap. Fig. 22 shows how the focusing (attractive) nonlinearity leads to a conversion of the pulse width, controlled by both nonlinearity and dispersion, remains unchanged at least on the timescale of our calculations. A special attention to formation of a bright soliton from the emitted blip and its stability was given in our previous paper [29. In the two other cases, the propagating blip starts exhibiting oscillatory tails typical of the dispersive shock waves $34 / 35 / 36 / 37$. These oscillations are visibly stronger for the defocusing case. The shock waves are governed by the dispersion and by defocusing media (repulsive interaction in BEC). 


\subsection{Pulsations inside the trap}

An interesting result is that the initial packet wider than the trap very rapidly decays into a narrow packet loosely related to the ground state inside the trap with high shoulders outside the trap. This early time evolution takes place for all packets whose initial width is wider than the trap.

As described above the initial packet emits a pair of blips and forms a rather narrow packet inside the potential well, which is rather close to the ground state of the well, obtained when disregarding the tunneling. This narrow packet is a state with a long life time which does not essentially decay during our simulation time. However, we observe well pronounced pulsations of the narrow packet with roughly doubled trap eigen frequency. These pulsations are seen in Figs. 22 and are shown in the 3-d plots in Fig. (3). We also see that in the focusing case, the oscillations have higher frequency and the latter increases as the nonlinearity

increases, while in the defocusing case (repulsive interaction in BEC) the pulsations are of lower frequencies.

In order to analyze the pulsations within the trap we continue along the lines of the hydrodynamic approach whose application to the tunneling problem was presented in our previous paper 29. The NLSE/GPE for the complex function can be rewritten as two equations for two real functions: the continuity equation

$$
\rho_{t}(x, t)+[\rho(x, t) v(x, t)]_{x}=0 .
$$

for the particle density distribution $\rho(x, t)=|\Psi(x, t)|^{2}$ and the Euler-type equation

$$
v_{t}(x, t)+v(x, t) v_{x}(x, t)=-\frac{1}{m} \nabla_{x}\left[V_{e x t}(x)-\frac{1}{\sqrt{\rho(x, t)}} \frac{\hbar^{2}}{2 m} \nabla_{x}^{2} \sqrt{\rho(x, t)}+\lambda \rho(x, t)\right] .
$$

for the velocity field $v(x, t)=-\nabla_{x} \varphi(x, t)$ with $\varphi(x, t)$ being the phase of $\Psi(x, t)$.

We assume the harmonic approximation

$$
V_{\text {ext }}(x)=\frac{m \nu^{2} x^{2}}{2}
$$

for the shape of potential inside the trap and for the time being neglect the nonlinearity. The density of narrow packet formed after the emission of blips is assumed to have a Gaussian shape

$$
\rho_{0}(x)=\frac{N}{u_{0} \sqrt{\pi}} \exp \left\{-\frac{x^{2}}{u_{0}^{2}}\right\}
$$

where $u_{0}$ does not necessarily coincide with the amplitude of zero point oscillations $u_{\text {ext }}=$ $\sqrt{\hbar / m \nu}$ in the potential (91). It rather corresponds to another harmonic potential with the frequency $\omega=\hbar / m u_{0}^{2}$.

We look for the solution of eqs. (7) and (8) also in the Gaussian form

$$
\rho(x)=\frac{1}{f(t)} \rho_{0}\left(\frac{x}{f(t)}\right)
$$

and

$$
v(x, t)=x \frac{d}{d t} \ln f(t)
$$



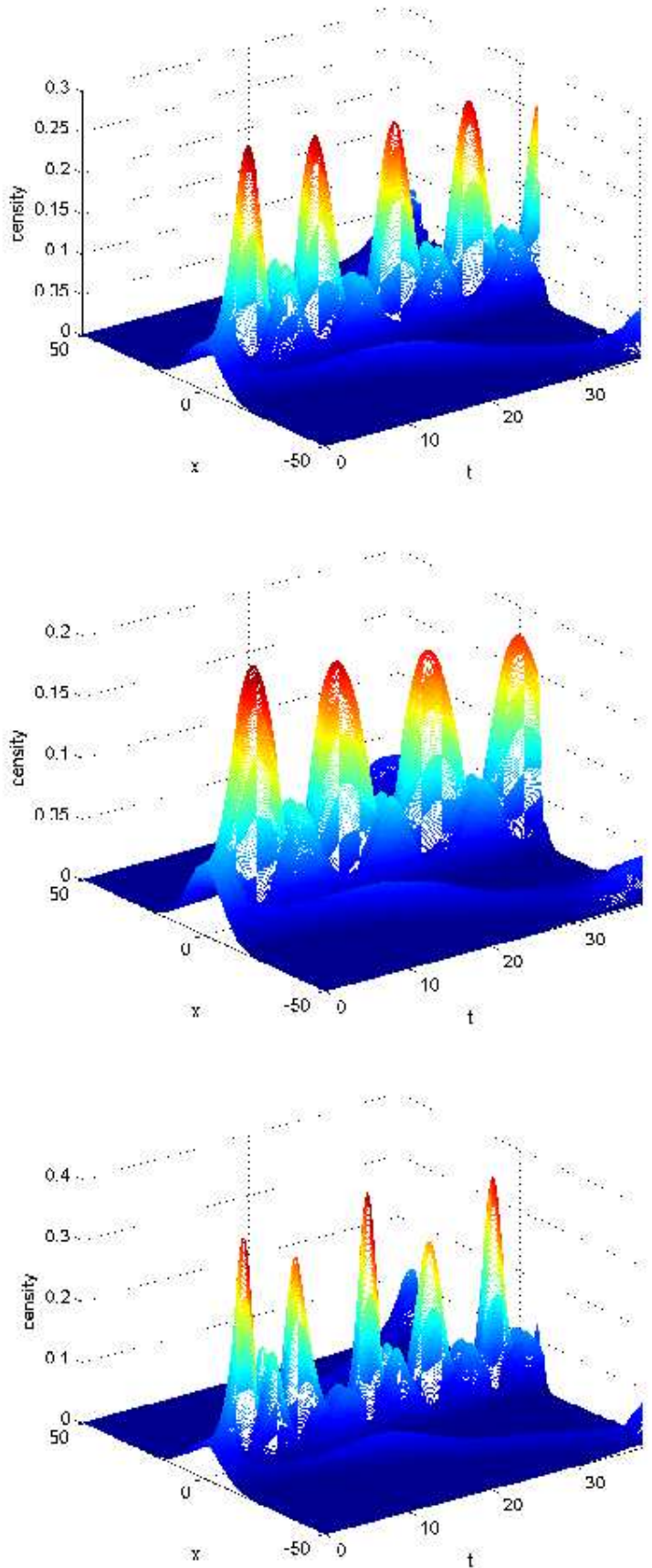

Fig. 3. Pulsations inside the trap after emission of a blip. From top left: zero, positive (repulsive/defocusing) and negative (attracting/focusing) nonlinearity. 
where $u(t)=u_{0} f(t)$ and $f(0)=1$. It means that the time dependence of the solution is parameterized by the single function $f(t)$. The functions (10) and (11) solve eq. (7) and when substituted into (8) for $\lambda=0$ yield equation

$$
f^{\prime \prime}=-\nu^{2} f+\omega^{2} f^{-3} .
$$

for the function $f(t)$. This is the equation of motion for a 'particle' with the unit mass and with the coordinate $f$ in the 'effective potential'

$$
W_{e f f}(f)=\nu^{2} \frac{f^{2}}{2}+\frac{\omega^{2}}{2 f^{2}} .
$$

The function $f(t)$ oscillates in this potential well between its initial value $f_{0}=f(0)=1$ (we assume also that $f^{\prime}(0)=0$ ) and the point $f_{1}=\omega / \nu$ found from energy conservation considerations. These two values of the function $f(t)$ correspond to the minimal and maximal widths of the pulsating Gaussian packet.

To find the full solution for $f(t)$ we note that eq. (12) can be mapped onto the equation of motion for a radially symmetric $2 \mathrm{~d}$ harmonic oscillator with the frequency $\nu$ in which $f$ is its radial coordinate. Then eq. (12) is obtained for the motion with the conserved angular momentum $\omega$.

The solution satisfying the above initial conditions is

$$
f(t)=\sqrt{\cos ^{2} \nu t+\frac{\omega^{2}}{\nu^{2}} \sin ^{2} \nu t .}
$$

which describes the dynamics of the width of the trapped packet.

It is interesting to indicate that there is a more general analytical solution

$$
\rho(x)=\frac{1}{f(t)} b^{2}\left(\frac{x}{f(t)}\right) \rho_{0}\left(\frac{x}{f(t)}\right)
$$

where

$$
b^{2}\left(\frac{x}{f(t)}\right)=C_{1}+i C_{2} f(t) \operatorname{erf}\left(\frac{i x}{u_{0} f(t)}\right)
$$

with the same parameterizing function $f(t)$ as in (10). The parameters $C_{1}$ and $C_{2}$ should be found from the normalization of the density distribution (15) and its initial shape. The error function in (16) is odd in $x$ which makes the distribution (15) asymmetric unless we assume that one of the parameters $C_{1}$ or $C_{2}$ zeros. If $C_{2}=0$ we return to the Gaussian distribution considered above. If, however, $C_{2}=0$ we have a symmetric distribution with a minimum at $x=0$ similar to what we see at the maximum amplitude of the pulsations.

In the presence of nonlinearity $(\lambda \neq 0)$ we cannot get an exact solution, however we may consider eqs. (10) and (11) as an approximate trial functions and find the energy of the pulsating packet in units $\hbar \nu$,

$$
\varepsilon_{d r o p}=\frac{1}{2} \dot{f}^{2}+W_{e f f}(f)
$$

where the first term in the r.h.s. stands for the kinetic energy of the pulsations. The second term

$$
W_{e f f}(f)=\nu^{2} \frac{f^{2}}{2}+\omega^{2} \frac{1}{2 f^{2}}+\frac{\lambda N}{f \sqrt{2 \pi}}
$$




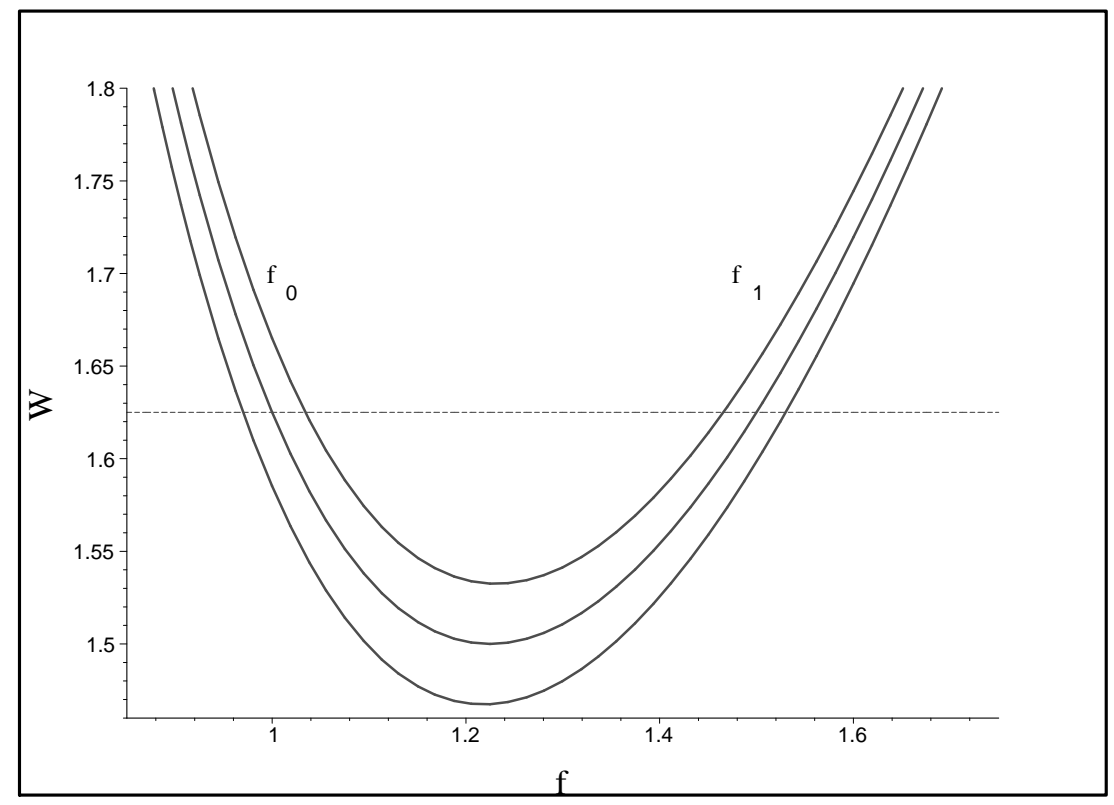

Fig. 4. The effective potential (18) for various interaction parameters: $N \lambda / \nu^{2}=0.1$ upper curve, $N \lambda / \nu^{2}=0-$ middle curve, $N \lambda / \nu^{2}=-0.1$ - lower curve. $\omega / \nu=1.5$. The horizontal line shows the level of oscillations between the points $f_{0}$ and $f_{1}$ in the case of $\lambda=0$.

represents the new effective potential (instead of (13)), which is just the internal energy of the packet at the given radius $f u_{0}$ obtained by averaging the BEC hamiltonian (1) with the above trial function. It means that the oscillations of the packet are governed by the equation of motion

$$
f^{\prime \prime}=-\nu^{2} f+\omega^{2} f^{-3}+\frac{\lambda N}{f^{2} \sqrt{2 \pi}}
$$

Fig. 4 presents the effective potentials for three values of the dimensionless nonlinearity $N \lambda / \nu^{2}$. We see in this figure that the repulsive interaction decreases the minimal width and increases the maximal width, i.e. it increases the amplitude of pulsations. A more detailed analysis shows that the period of pulsations also increases - the frequency lowers. The attractive interaction acts in the opposite direction and the frequency grows. This type of behavior agrees with the simulation results which can be clearly seen from Fig. 3 - the number of peaks in the same time interval varies for the three graphs corresponding to the different values of the interaction parameter.

The graph in fig. 3 for the nonfocusing case (zero interaction) shows that the period is roughly $T=8$. To estimate the trap frequency, which is nearly harmonic near the center of the well, we fit it to a parabolic function from which we deduce the frequency to be around $\nu_{\text {trap }} \simeq 0.0737$. Therefore the expected period would be $T=\pi / \nu_{\text {trap }} \simeq 8.18$ in a very good agreement with the theoretical prediction.

The simulation results for the NLSE, eq. (3), show that the fraction of the initial pulse which stays trapped along the propagating axis, exhibits pulsations of period $\sim 5000 \mathrm{~m} \mu$. To discuss the frequency equivalent for light propagation we look at the second term in eq. (3). Taking only the inside part of the trap and removing the constant offset, this term can be rewritten as 


$$
\sim-\left(\frac{2 \pi}{\lambda}\right)^{2} \cdot n \cdot \Delta n_{\max } \cdot\left(\frac{x}{100}\right)^{2}
$$

and is to be fitted to the harmonic potential $\nu^{2} x^{2} / 2$ for trapped particles. Then we get $T=\pi / \nu=7200 \mu \mathrm{m}$ for 'the spatial period', which is close to the simulation result $T_{\text {sim }} \sim$ $5000 \mu \mathrm{m}$.

It is worth mentioning here that various type of oscillations have been discussed in literature (see, review [16]) as a rule using the Thomas-Fermi approximation which neglects the quantum pressure term. This approximation works especially well for small amplitude oscillations with the repulsive interaction when the density distribution is rather close to uniform. It is emphasized here that the density distribution in the tunneling problem we discuss here is strongly nonuniform and the pulsations discussed here are strong so that the role of the quantum pressure is of an utter importance.

\section{Atomic Pulsed Laser}

Combining the two discussed effects, i.e. blip emission due to macroscopic tunneling and in-trap periodic pulsations, one can propose a new mechanism of controllable multiple blip emission. Such effect may lead to a realization of Atomic Pulsed Laser. The mechanism we propose is based on narrowing the well to a desired width, according to eq. (13) at times that match the pulsating packet being at its maximum width and allows for outcoupling matter waves pulses. In addition to the simple realization, a full control and modulation possibility of several solitonic features, also in the course of emission, is at hand. In order to demonstrate this we consider the potential

$$
U_{e x t}=C(t) \frac{x^{2}}{\cosh ^{2}\left(\frac{x}{a(t)}\right)} .
$$

with the varying in time parameters

$$
a(t), C(t)= \begin{cases}a_{1}, C_{1} & t<t_{1} \\ a_{2}, C_{2} & t<t_{2} \\ \cdots & \end{cases}
$$

Variations of $a(t)$ and $C(t)$ are fitted in such a way as to keep the height of the barrier unaltered if identical velocities of the pulses are desired. Alternatively they may be chosen to vary the barrier height in order to reach the desired variation of the velocities of outcoupled pulses. $a(t)$ is the variable width of the well. The times $t_{1}, t_{2} \ldots$ are set to match the period of pulsations. It is important to stress here that the latter changes according to the width of the narrowing trap as was proved earlier. Therefore, on-switch times alter accordingly.

Three pairs of blips emitted from the trap are seen in fig. 5 . The first pair of blips is emitted at the start of the process and the two other pairs are emitted due the two narrowings at $t=8$ and $t=13$ with the widths $a=5,4$ and 0.7 respectively. Since the nonlinearity plays a minor role in the emission process this simulation is carried out without it.

Velocity modulation. As stated above, our analysis, based on the hydrodynamic approach, predicts a one to one relation between the velocity and the height of the barriers, 


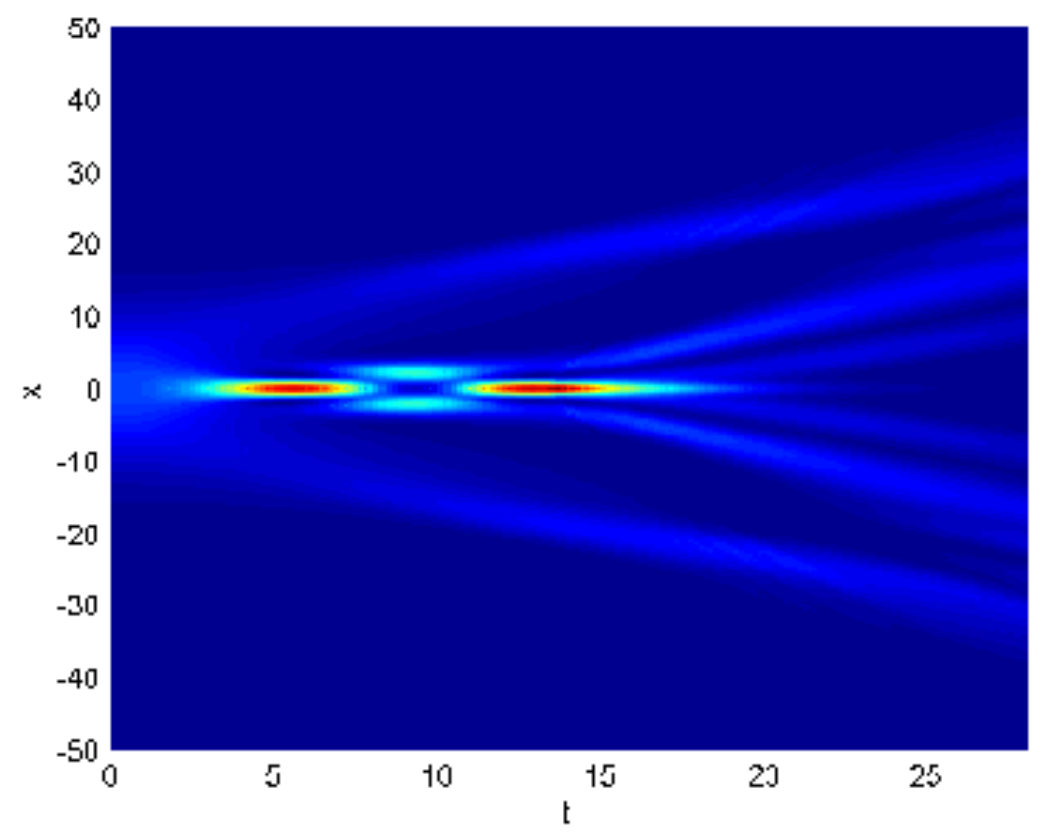

Fig. 5. Emission of the second and third pairs of blips by the trap twice subsequently squeezed at the maximal width of pulsations.

leading to a viable control of the outcoupled pulse velocities also in the course of sequential emissions. In this case the velocity of the blips is altered according to

$$
v_{\text {blips }}=\sqrt{2 C(t) s(t)}
$$

where $s(t)$ is the solution of the transcendental equation

$$
s=\frac{\tanh (a(t) s)}{a(t)}
$$

Fig. 6 shows the velocity variation for a single pair of pulses during its propagation, and for two pairs of pulses which are made to propagate with different velocities.

The velocity difference between the two consecutive outcoupled pulses has been created by temporally changing the barrier height. The restriction here being that the first propagating outcoupled pulse has to be far enough from the barrier region, so that it will not be effected as well.

Temporal interval modulation and control Temporal spacing modulation can be achieved by considering different numbers of pulsation periods between two emissions. Another feature that should be taken into consideration is the time of the blip formation. This time is shown in ref. 29] to vary according to the trap parameters as

$$
t_{b} \sim\left(\frac{8 U_{0}}{a^{2}}\right)^{-\frac{1}{2}}
$$

Therefore the formation time becomes shorter for the narrower barriers. 

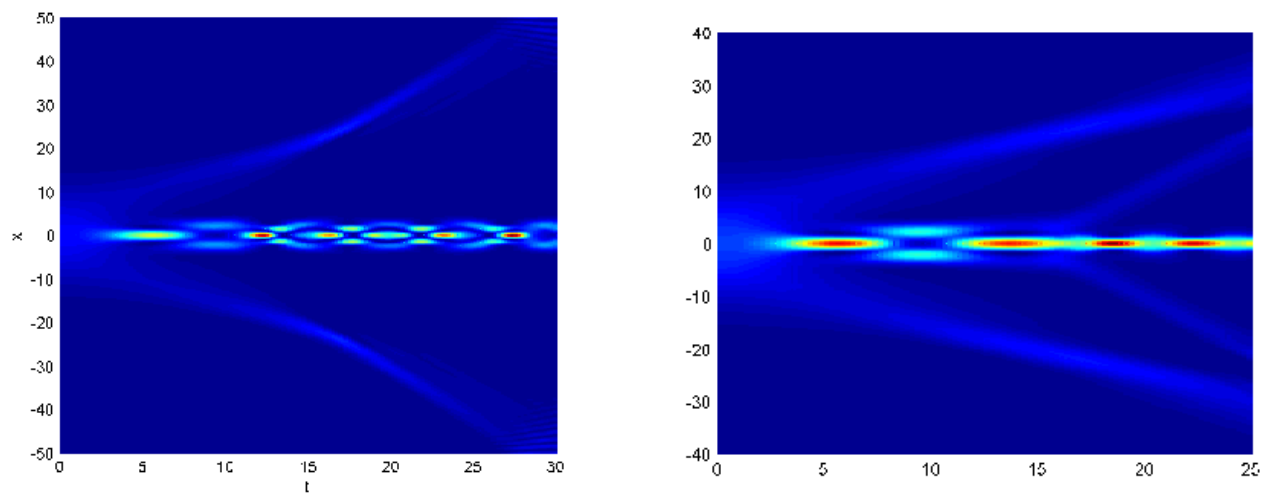

Fig. 6. Velocity modulation achieved by raising the barrier height. Left: modulation of single pulse velocity, during its propagation. At $t=15$ the barrier peak is raised from $\simeq 1$ to $\simeq 4$ Right: production of two consecutive pairs of pulses with different velocities. At $t=15$ the trap is narrowed from $\mathrm{a}=5$ to $\mathrm{a}=2.5$ and the barrier peak is raised from $\simeq 1$ to $\simeq 2$

Stability The pulse creation is not related to the nonlinearity of the GPE and the present discussion without loss of generality is limited to the zero interaction. However, it is of course evident that for an operating pulsed laser the pulses have to transform into bright solitons and moreover need to be kept stable and not disperse. This can be achieved by turning on attractive interaction along the way with lower limit according to the soliton formation condition

$$
U_{\text {int }} \cdot|\psi(0, T)|^{2} \sim 6.5 a^{-2}+0.03 U_{0}
$$

found in ref. 22] for the shape of trap potential which is rather close to the trap chosen here. In fact the parameter values in eq. (25) may slightly change with the shape of the particular trap. Here $T$ is the on-set time. $|\psi(0, T)|^{2}$ the density in the center of the trap at $t=T$. If the condition 25 is satisfied, the emerging pulse may transform into a stable bright soliton. In $2 \mathrm{~d}$ and $3 \mathrm{~d}$ case some stricter conditions as say Vakhitov-Kolokolov 41 should be satisfied. Since the emerging pulses are much more dilute than the initial packet, stability against collapse in $2 \mathrm{~d}$ and $3 \mathrm{~d}$ case is easier achievable. The masses and widths of the pulses can be also found as described for the single emission event in ref. 29].

In principle we can consider restoring the original width of the trap after each emission and repeat this process as many times as necessary. This mechanism will work as long as there is matter left within the trap and its coherency is held.

\section{Conclusions}

Certain aspects of the universality of the emission of blips in the course of macroscopic tunneling were asserted, using two simulation programs, one of which applying directly to Optics. Pulsations of the trapped packet after emergence of the blips were shown by the hydrodynamic approach and by numerics to occur with the period matching twice the trap frequency, which inspired us to suggest a mechanism for realization of Atom Pulsed Laser. The blips of the matter density may be emitted by controllably narrowing the trap. The 
velocity of the outgoing pulses may be controlled by varying the height of the barrier. We show also how to control the times of the pulse emissions and their masses.

Acknowledgments. The authors acknowledge the support of United States - Israel Binational Science Foundation, Grant N 2006242 and of Israeli Science Foundation, Grant N 944/05. The authors are also grateful to Max Planck Institute for Physics of Complex Systems, Dresden, for hospitality. We are grateful to S. Bar-Ad, Y. Linson, and M. Weitz for help and stimulating discussions.

\section{References}

[1] S. Burger, K. Bongs, S. Dettmer, W. Ertmer, K. Sengstock, Phys. Rev. Lett., 83, 5198 (1999).

[2] J. Denschlag, J.E. Simsarian, D.L. Feder, C.W. Clark, L.A. Collins, J. Cubizolles, L. Deng, E.W. Hagley, K. Helmerson, W.P. Reinhardt, S.L. Rolston, B.I. Schneider, W.D. Phillips, Science, 287, 97 (2000).

[3] L. Khaykovich, F. Schreck, G. Ferrari, T. Bourdel, J. Cubizolles, L.D. Carr, Castin, Y., C. Salomon, Science 296, 1290 (2002).

[4] K.E. Strecker, G.B. Partridge, A.G. Truscott, R.G. Hulet, Nature (London) 417, 150 (2002).

[5] M.O. Mewes, M.R. Andrews, D.M. Kurn, D.S. Durfee, C.G. Townsend, W. Ketterle, Phys. Rev. Lett., 78, $582(1997)$.

[6] B.P. Anderson, and M.A. Kasevich, Science, 282, 1686 (1998).

[7] I. Bloch, T.W. Hansch, and T. Esslinger, Phys. Rev. Lett., 82, 3008 (1999).

[8] E.W. Hagley, L. Deng, M. Kozuma, J. Wen, K. Helmerson, S.L. Rolston, W.D. Phillips, Science, 283, 1706 (1999).

[9] J.L. Martin, C.R. McKenzie, N.R. Thomas, J.C. Sharpe, D.M. Warrington, P.J. Manson, W.J. Sandle, A.C. Wilson, J. Phys. B, 32, 3065 (1999).

[10] M.I. Rodas-Verde, H. Michinel, V.M. Pérez García, Phys. Rev. Lett., 95, 153903 (2005).

[11] A.V. Carpentier, H. Michinel, M.I. Rodas-Verde, V.M. Pérez García, Phys. Rev. A, 74, 013619 (2006).

[12] L.D. Carr, J. Brand, Phys. Rev. A, 70, 033607 (2004).

[13] P.Y.Y Chen, B.A. Malomed, J. Phys. B: At. Mol. Opt. Phys. 384221 (2005).

[14] P.Y.Y Chen, B.A. Malomed, J. Phys. B.: At. Mol. Opt. Phys. 392803 (2006).

[15] A.L. Fetter, Phys. Rev. Lett., 138, A429 (1965).

[16] F. Dalfovo, S. Giorgini, L.P. Pitaevskii, S. Stringari, Rev. Mod. Phys. 71, 463 (1999).

[17] E.A. Ostrovskaya, Y.S. Kivshar, M. Lisak, B. Hall, F. Cattani, D. Anderson, Phys. Rev. Lett. 61, 031601 (2000).

[18] L.D. Carr, M.J. Holland, B.A. Malomed, J.Phys B 38, 3217 (2005).

[19] N.Moiseyev, L.D. Carr, B.A. Malomed, and Y.B. Band, J. Phys. B 37, L193 (2004).

[20] S. Osovski and N. Moiseyev, Phys. Rev. A 72, 033603 (2005)

[21] E. Madelung, Z. Phys. 40, 322 (1927).

[22] J.H. Marburger, Prog. Quantum Electron. 4, 35 (1975).

[23] Y. Silberberg, Opt. Lett. 15, 1282 (1990).

[24] M.A. Hoefer, M.J. Ablowitz, I. Coddington, E.A. Cornell, P. Engels, and V. Schweikhard, Phys. Rev. A 74, 023623 (2006).

[25] P. R. Holland, 1993 The Quantum Theory of Motion Cambridge Univ. Press

[26] S. Stringani, Phys. Rev. Lett. 77, 2360 (1996).

[27] M. Levanda and V. Fleurov, Annals of Physics, 292, 199 (2001)

[28] V. Fleurov and A. Soffer, Europhys.Lett., 72, 287 (2005).

[29] G. Dekel, V. Fleurov, A. Soffer, C. Stucchio, Phys. Rev. A., 75, 043617 (2007).

[30] Ph. Balcou, L. Dutriaux, Phys. Rev. Lett., 78, 851 (1997).

[31] E. Pollak, W.H. Miller, Phys, Rev. Lett., 53, 116 (1984).

[32] M. Büttiker, R. Landauer, Phys. Rev. Lett., 49, 1740 (1982).

[33] E.H. Hauge, J.A. Støvneng, Rev. Mod. Phys., 61, 917 (1989).

[34] M.A. Hoeffer, M.J. Ablowitz, I. Coddington, E.A. Cornell, P. Engels, V. Schweikhard, Phys. Rev. A., 74, 023623 (2006). 
[35] B. Damski, Phys. Rev. A., 69, 043610 (2004).

[36] A.M. Kamchatnov, A. Gammal, R.A. Kraenkel, Phys. Rev. A., 69, 063605 (2004).

[37] G.A. El, A.M. Kamchatnov, Phys. Lett. A., 350, 192 (2006).

[38] Wenjie Wan, Shu Jia and J. W. Fleischer, Nature Physics 3, 46 - 51 (2007).

[39] R.J. Ballagh, Computation methods for solving nonlinear partial differential equations. University of Otago (2000).

[40] The program was prepared by H. Eisenberg, Hebrew University of Jerusalem.

[41] N. G. Vakhitov and A. A. Kolokolov, Izv. Vyssh. Uch. Zaved., Radiofizika 16, 10120 (1973) [Radiophys. Quantum Electron. 16, 783 (1973)] 